\section{Band 17, Heft 5, Oktober 2010}

\section{Editorial}

237 Gestresste Patienten, getresste Ärzte und der Bedarf an Mind-Body-Medizin

Michalsen, A. (Berlin)

\section{Übersichtsarbeit}

241 Chelidonium majus - eine integrative Übersicht: Traditionelles Wissen versus moderne Erkenntnisse Gilca, M.; Gaman, L.; Panait, E.; Stoian, I.; Atanasiu, V. (Bucharest)

Originalarbeiten

250 Effekte homöopathischer Medikamente auf die Stimmung von Erwachsenen mit Kaffee-induzierter Schlaflosigkeit in der Anamnese

Brooks, A.J.; Bell, I.R.; Howerter, A.; Jackson, N.; Aickin, M. (Tucson, AZ)

259 Sind Scheinakupunktur-Interventionen wirksamer als (andere) Placebos? Eine Reanalyse von Daten aus dem Cochrane-Review zu Placeboeffekten Linde, K.; Niemann, K.; Meissner, K. (München)

\section{Kurzmitteilung}

266 Können in der Behandlung von venösen Geschwüren pflanzliche Therapien mit physikalische Maßnahmen kombiniert werden? Bewertung des Heilprozesses und der antimikrobiologischen Wirkung: Eine Pilotstudie Janković, A.; Binić, I. (Niš); Vručinić, Z. (Banja Luka); Janković, D. (Kragujevac); Janković, I. (Niš); Jančić, S. (Kragujevac)

\section{Falldarstellung}

271 Topische Behandlung eines nekrotisierenden Herpes zoster mit Betulin aus der Birkenrinde

Weckesser, S. (Freiburg i.Br.); Laszczyk, M.N. (Darmstadt); Müller, M.L.; Schempp, C.M.; Schumann, H. (Freiburg i.Br.)

\section{Vol. 17, Issue 5, October 2010}

Editorial

237 Stressed Patients, Stressed Physicians and the Need for Mind-Body Medicine

Michalsen, A. (Berlin)

Review Article

241 Chelidonium majus - an Integrative Review: Traditional Knowledge versus Modern Findings Gilca, M.; Gaman, L.; Panait, E.; Stoian, I.; Atanasiu, V. (Bucharest)

Original Articles

250 Effects of Homeopathic Medicines on Mood of Adults with Histories of Coffee-Related Insomnia Brooks, A.J.; Bell, I.R.; Howerter, A.; Jackson, N.; Aickin, M. (Tucson, AZ)

259 Are Sham Acupuncture Interventions More Effective than (Other) Placebos? A Re-Analysis of Data from the Cochrane Review on Placebo Effects Linde, K.; Niemann, K.; Meissner, K. (München)

\section{Short Communication}

266 Can You Combine Herbal Therapy with Physical Agents in the Treatment of Venous Leg Ulcers? Evaluation of Healing and Antimicrobiological Effects: A Pilot Study

Janković, A.; Binić, I. (Niš); Vručinić, Z. (Banja Luka); Janković, D. (Kragujevac); Janković, I. (Niš); Jančić, S. (Kragujevac)

Case Report

271 Topical Treatment of Necrotising Herpes Zoster with Betulin from Birch Bark

Weckesser, S. (Freiburg i.Br.); Laszczyk, M.N. (Darmstadt); Müller, M.L.; Schempp, C.M.; Schumann, H. (Freiburg i.Br.) 


\section{Band 17, Heft 5, Oktober 2010}

Dokumentation

275 Forschungsinitiativen in Europa

Robinson, N. (Brentford); Güthlin, C. (Frankfurt/M.);

Weidenhammer, W. (München); Wolf, U. (Bern)

Brief an die Herausgeber

277 Personzentrierte Medizin: Versuch einer Definition Roberti di Sarsina, P. (Rom); Iseppato, I. (Bologna)

279 Journal Club

279 Effekte der Arzt-Patient-Kommunikation in der Akupunktur

281 Der spezifische Effekt von Akupunktur bei peripherer Gelenksarthrose - statistisch signifikant, aber klinisch irrelevant?

282 Ist Massage bei generalisierter Angststörung wirksam?

283 Massage kann sinnvoll bei Depression sein

Abstract Service

286 Latest Publications You Should Not Miss

Gesellschaftsmitteilungen

289 Schweizerische Medizinische Gesellschaft für Phytotherapie (SMGP)

292 News / Ticker

293 Tagungen und Kongresse

295 Hinweise für Autoren

234 Impressum
Vol. 17, Issue 5, October 2010

Documentation

275 Research Initiatives in Europe

Robinson, N. (Brentford); Güthlin, C. (Frankfurt/M.);

Weidenhammer, W. (München); Wolf, U. (Bern)

Letter to the Editors

277 Person-Centred Medicine: Towards a Definition Roberti di Sarsina, P. (Rom); Iseppato, I. (Bologna)

279 Journal Club

279 On the Effect of Patient-Provider Vommunication in Acupuncture

281 The Specific Effect of Acupuncture for Peripheral Joint Osteoarthritis, Statistically Significant but Not Clinically Relevant?

282 Is Massage Effective for Generalized Anxiety Disorder?

283 Massage Therapy Can Be Helpful for Depression

Abstract Service

286 Latest Publications You Should Not Miss

Society Bulletins

289 Schweizerische Medizinische Gesellschaft für Phytotherapie (SMGP)

292 News / Ticker

293 Meetings and Conferences

295 Guidelines for Authors

234 Imprint
Einen Ausblick auf den Inhalt der kommenden Hefte finden Sie auf Seite 296.
Forthcoming papers are listed on page 296. 